\title{
Use of Fibrates in Clinical Practice: Queensland Lipid Group Consensus Recommendations
}

\section{Summary}

Fibrates have been prescribed for decades as 'broad-spectrum' lipid modifying agents that can improve plasma levels of triglycerides (TGs), highdensity lipoprotein cholesterol (HDL-C), and triglyceride-rich lipoproteins (TGRL), including very low- and intermediate-density lipoproteins (VLDL and IDL). Fibrates are variably effective in lowering LDL cholesterol (LDL-C) levels. Available fibrates include gemfibrozil, fenofibrate, bezafibrate, etiofibrate and ciprofobrate; only fenofibrate and gemfibrozil are available in Australia. Fibrates are well tolerated, and the combination of fenofibrate with statins appears to be safer than gemfibrozil, particularly with regard to adverse effects on muscle. Evidence has been provided recently for the efficacy of fenofibrate in reducing microvascular complications in diabetic patients, including progression of retinopathy, progression of microalbuminuria and nephropathy, development of sensory neuropathy, and leg amputation. Macrovascular benefits appear to be confined to those with low HDL-C and/or high TG levels, and the relationship of microvascular benefits of fenofibrate to baseline lipid levels is variable and requires further assessment. Indications for fibrate therapy may be extended in the future to include protection from both macro- and micro-vascular disease, particularly in diabetic patients and patients with residual dyslipidaemia in spite of statin 
therapy. We provide recommendations on the use of fibrates in clinical practice to highlight these potential indications.

\section{Background}

Fibrates are peroxisome proliferator activator receptor (PPAR)-alpha agonists, thereby activating several genes involved in lipid metabolism, the result of which includes increased fatty acid oxidation, decreased hepatic incorporation of fatty acids into VLDL and reduced VLDL production (1-9). In parallel, reduced synthesis and plasma levels of apo C-3 activates lipoprotein lipase (LPL), which increases VLDL catabolism, reduces plasma concentration of large VLDL particles, and lowers plasma levels of TG. Lowering the plasma TG level is the main indication for fibrate therapy. With TG reduction, numbers of atherogenic small, dense LDL particles decrease, and numbers of less atherogenic large, less dense LDL particles increase. Potentially beneficial changes in the size distribution of LDL particles may therefore occur without change in the overall LDL cholesterol (LDL-C) concentration. Plasma concentrations of HDL and HDL-C levels also increase, partly originating from surface components of VLDL during hydolysis, and partly from increased Apo A-1 synthesis and activation of $A B C-A 1$ cassette transporters, with activation of reverse cholesterol transport (RCT). Fibrates also decrease plasma uric acid and have anti-inflammatory properties through decreased levels of interleukin-6 (IL-6), fibrinogen, cell-adhesion molecules and C-reactive protein $(\mathrm{CRP})$. These properties are consistent with anti-atherogenic effects on lipid metabolism. Fenofibrate, unlike gemfibrozil, increases serum homocysteine, creatinine and cystatin. In patients with chronic kidney disease 
(CKD), the dose of fenofibrate needs to be reduced, unlike that of gemfibrozil, as there is an increase in plasma levels of the drug and consequently an increased risk of side effects at usual dose (1-9).

\section{Aims}

This paper reviews evidence for the benefits of fibrates in controlling dyslipidaemia, and preventing both macrovascular cardiovascular disease (CVD) and diabetic microvascular disease. It provides recommendations for the use of fibrates in clinical practice in the context of statin therapy for controlling levels of LDL-C, which remains the primary focus of therapy for preventing CVD. It supplements recent guidelines on the use of fibrates as well as reviews on TG as CVD risk factors (1-19)

\section{Methods}

Data presented herein are derived from PubMed searches, product information of fibrates, and personal clinical experience of members of the Queensland Lipid Group. Grades of recommendations (Table 1) were applied to each of the recommendations in the manuscript, reflecting the consensus of the Group as a panel of experts that met at workshops in Brisbane during 2010-11 (20). Members of the panel were asked to grade the recommendations based on review of the literature and experience in treating patients with fibrates. We intended to keep the grades simple and driven by what was considered best practice. A hierarchical analysis of the evidence was not employed to avoid giving a low grade to recommendations that were considered best clinical practice and to offset confusion, especially where 
systematic reviews and randomized controlled were not available for essential practice points.

Table1. Grades of recommendations employed for consensus recommendations.

\begin{tabular}{|c|c|}
\hline $\begin{array}{l}\text { Grade of } \\
\text { Recommendation }\end{array}$ & Description \\
\hline $\mathbf{A}$ & $\begin{array}{l}\text { Recommendation can be trusted to guide } \\
\text { practice }\end{array}$ \\
\hline B & $\begin{array}{l}\text { Recommendation can be trusted to guide } \\
\text { practice in most situations }\end{array}$ \\
\hline C & $\begin{array}{l}\text { Recommendations may be used to guide } \\
\text { practice but care should be taken in its } \\
\text { application }\end{array}$ \\
\hline
\end{tabular}

\section{Results}

As a result of the panel meetings of the Queensland Lipid Group, the following recommendations and consensus grades are made on the use of fibrates in clinical practice (Table 2).

Table 2. Recommendations on the use of fibrates in clinical practice and consensus grade. 


\begin{tabular}{|c|c|c|}
\hline No. & Components & Grade \\
\hline 1 & $\begin{array}{l}\text { Fibrates are first-line therapy for improving the lipid profile } \\
\text { in patients with fasting TG levels of } 4.5 \mathrm{mmol} / \mathrm{L} \text { or more, } \\
\text { assuming adequate glycaemic control in diabetic patients } \\
\text { and exclusion of other secondary causes of } \\
\text { dyslipidaemia }\end{array}$ & $A$ \\
\hline 2 & $\begin{array}{l}\text { Fibrates are second-line therapy for patients with } \\
\text { elevated fasting TG levels less than } 4.5 \mathrm{mmol} / \mathrm{L} \text {, } \\
\text { assuming adequate glycaemic control in diabetic patients } \\
\text { and exclusion of other secondary causes of } \\
\text { dyslipidaemia }\end{array}$ & $A$ \\
\hline 3 & $\begin{array}{l}\text { Fibrate monotherapy may reduce LDL-C levels to a } \\
\text { variable degree, but fibrates are not generally used for } \\
\text { this purpose. Fenofibrate may be considered for patients } \\
\text { on statin therapy with elevated triglycerides. For further } \\
\text { LDL-C reduction, alternative agents to consider include } \\
\text { plant sterols, ezetimibe, bile resins and nicotinic acid. }\end{array}$ & $A$ \\
\hline 4 & $\begin{array}{l}\text { Fenofibrate is preferred to gemfibrozil for combination } \\
\text { therapy with statins in order to reduce the likelihood of } \\
\text { adverse effects on skeletal muscle }\end{array}$ & $A$ \\
\hline 5 & $\begin{array}{l}\text { Fibrates are appropriate for prevention of macrovascular } \\
\text { cardiovascular disease in patients with high fasting TG } \\
\text { levels ( } 2.3 \mathrm{mmol} / \mathrm{L} \text { or more) and/or low HDL-C levels } \\
\text { (less than } 1.0 \mathrm{mmol} / \mathrm{L} \text { in men and less than } 1.2 \mathrm{mmol} / \mathrm{L} \text { in }\end{array}$ & B \\
\hline
\end{tabular}




\begin{tabular}{|l|l|l|}
\hline & $\begin{array}{l}\text { women), assuming adequate glycaemic control in } \\
\text { diabetic patients and exclusion of other secondary } \\
\text { causes of dyslipidaemia }\end{array}$ & \\
\hline 6 & $\begin{array}{l}\text { Fenofibrate may be considered for prevention of } \\
\text { microvascular disease (retinopathy, sensory neuropathy, } \\
\text { nephropathy and to prevent amputations) in patients with } \\
\text { type } 2 \text { diabetes mellitus, assuming adequate glycaemic } \\
\text { control and exclusion of other secondary causes of } \\
\text { dyslipidaemia }\end{array}$ & \\
\hline
\end{tabular}

\section{Discussion}

Recommendation 1 (Grade A). Fibrates are first-line therapy for improving the lipid profile in patients with fasting TG levels of 4.5 $\mathrm{mmol} / \mathrm{L}$ or more, assuming adequate glycaemic control in diabetic patients and exclusion of other secondary causes of dyslipidaemia.

Fibrates are effective therapy for moderate (plasma TG levels of 4.5-11.0 $\mathrm{mmol} / \mathrm{L}$ ) and severe HTG (plasma TG levels $>11.0 \mathrm{mmol} / \mathrm{L}$ ), often lowering plasma TG levels by $>50 \%(6-17)$. These conditions most commonly occur in association with uncontrolled diabetes mellitus or excessive alcohol consumption, especially binge drinking. Less frequent causes include hypothyroidism, renal disease, and excessive consumption of refined carbohydrates or fats. Drug therapy is often required in these conditions (Table 4) (6-17). Familial causes include familial HTG, familial dysbetalipoproteinaemia (type III hyperlipidaemia due to apoE2 
homozygosity) and the rare disorders involving LPL, apoC2, apoAV, apoAIV, and GPIHBP1 mutations. These genetic disorders are resistant to fibrates or other drug therapy. The major complication of severe HTG is recurrent acute pancreatitis, in contrast to moderate HTG, the major complication of which is CVD (Table 3) (10-14).

Table 3. Causes of hypertriglyceridaemia

Diabetes mellitus (uncontrolled)

Alcohol excess

Exogenous oestrogen (oral contraceptive, hormone replacement therapy)

Endogenous oestrogen (pregnancy)

Hypothyroidism

Severe proteinuria (nephrotic syndrome)

Excessive consumption of refined carbohydrates

Inadequate fasting before blood sampling (sampling during post-prandial lipaemia)

Multiple myeloma

Drugs: amiodarone, alpha-interferons, beta-blockers, bile acid resins, Lasparaginase, protease inhibitors, sirolimus, tamoxifen, thiazides, steroids, retinoic acids, atypical antipsychotics

Familial hypertriglyceridaemia

Familial combined hyperlipidaemia

ApoE2 homozygosity

ApoAV heterozygosity

ApoAIV homozygosity 
ApoC2 deficiency

GPIHBP1 mutations

Lipoprotein lipase deficiency

Fibrates reduce TG levels by enhancing the catabolism of fatty acids, thereby reducing the incorporation of TG into secreted VLDL, and by increasing the action of LPL and lipolysis of circulating TGRL (1-9).

In severe HTG, plasma viscosity is also reduced by fibrate therapy, a mechanism that may also reduce the risk of acute pancreatitis. Management of patient with severe HTG aims to maintain target TG levels sufficient to prevent acute pancreatitis - usually $<11 \mathrm{mmol} / \mathrm{L}$ (although lower levels may be required in some patients). In moderate HTG, target TG levels are $<1.5$ $\mathrm{mmol} / \mathrm{L}$, which will often require fibrate therapy in combination with statins, niacin, or omega-3 fatty acids.

According to the product information for fenofibrate, patients with moderate HTG (baseline TG 3.96-5.64 mmol/) achieve reductions of $46 \%(p<0.05$ vs placebo), $44 \%$ ( $p<0.05$ vs placebo) and $15 \%$ (NS vs placebo) in plasma levels of TG, total cholesterol (TC) and LDL-C respectively, and levels of HDL-C increase by $20 \% \quad(p<0.05$ vs placebo) (21). Patients with severe HTG (baseline TG 5.66-16.97 mmol/L) treated with fenofibrate achieve reductions of $55 \%, 51 \%$ and $49 \%$ in plasma levels of TG, total cholesterol (TC) and LDLC respectively (all $p<0.05$ vs placebo), and levels of HDL-C increase by $23 \%$ $(p<0.05$ vs placebo) $(21)$. 
High-dose n-3 fatty acids (EPA/DHA) at a dose of 2-4g daily lower TG levels with a similar efficacy to fibrates, and also augment TG lowering when added to fibrates (15). They can be considered as either alternative or add-on therapy to fibrates for patients with moderate-severe elevation of TG levels.

Recommendation 2, (Grade A). Fibrates may be considered as secondline therapy for patients with elevated fasting TG levels less than 4.5 $\mathrm{mmol} / \mathrm{L}$, assuming adequate glycaemic control in diabetic patients and exclusion of other secondary causes of dyslipidaemia.

Mild HTG is defined as elevated TG levels between 1.7 and $4.5 \mathrm{mmol} / \mathrm{L}$. After lifestyle modification, either fibrate or statin monotherapy may be effective in controlling TG levels in patients with mild HTG, and to achieve ideal TG levels $<1.5 \mathrm{mmol} / \mathrm{L}(1-9)$. We prefer statins as first-line therapy, however, because of their predictable effects on lowering levels of LDL-C, although moderately high doses of more potent statins may be required for TG control. In general, the efficacy of statin therapy in mild HTG depends on baseline TG (more effective with higher TG levels) and dose and potency of statins with regard to LDL-C reduction (more effective in higher doses and with increasing statin potency). Nevertheless, statin therapy alone may not achieve target levels of TG and combination statin-fenofibrate therapy may be required (1-9). The addition of omega-3 fatty acids (2-4g/day) may also assist in achieving target TG levels. As for patients with severe HTG, restriction of dietary refined carbohydrates, fats and alcohol is necessary, as is good glycaemic control in diabetic patients. 
Fibrates reduce levels of TG-rich remnant VLDL and IDL particles, as well as postprandial lipemia (15). These may augment the anti-atherosclerotic effects of increasing HDL-C levels (16).

Recommendation 3. Fibrate monotherapy may reduce LDL-C levels to a variable degree, but fibrates are not generally used for this purpose. Fenofibrate may be considered for patients on statin therapy with elevated triglycerides. For further LDL-C reduction, alternative agents to consider include plant sterols, ezetimibe, bile resins and nicotinic acid.

\section{Fenofibrate and baseline lipid levels}

The effect of Fenofibrate on LDL-C levels depends to a large extent on the baseline lipid profile. Patients with hypercholesterolemia alone may experience $10 \%$ to $20 \%$ reduction in LDL-C levels. In patients with borderline high and high TG, LDL-C levels are often unchanged, while LDL-C levels may increase in patients with very high TG.

Fibrate monotherapy in hypercholesterolaemia (HC)

Fibrates may be effective in patients who cannot tolerate statins, and whose LDL-C levels remain above target. Fibrates are alternative second-line therapy after ezetimibe for these patients, and niacin is the alternative second-line add-on therapy as discussed below.

A study of fenofibrate monotherapy in patients with severe hypercholesterolaemia and mildly elevated TG levels (Frederickson Type IIb) 
resulted in reductions of $35 \%, 14 \%$ and $14 \%$ in plasma levels of $\mathrm{TG}$, total cholesterol (TC) and LDL-C respectively (21). Levels of HDL-C were increased by $12 \%$ (all results $p<0.05$ vs. placebo). Baseline lipid levels in these patients $(\mathrm{N}=242)$ were: TC $8.1 \mathrm{mmol} / \mathrm{L}$, TG $2.6 \mathrm{mmol} / \mathrm{L}$, LDL-C 5.7 $\mathrm{mmol} / \mathrm{L}$ and HDL-C $1.2 \mathrm{mmol} / \mathrm{L}$. A similar study of fenofibrate monotherapy in patients with severe hypercholesterolaemia and normal TG levels (Frederickson Type Ila) resulted in reductions of $12 \%, 22 \%$ and $29 \%$ in plasma levels of TG, total cholesterol (TC) and LDL-C respectively (21). Levels of HDL-C were increased by $12 \%$ (all results $\mathrm{p}<0.05$ vs. placebo). Baseline lipid levels in these patients $(\mathrm{N}=334)$ were: TC $8.0 \mathrm{mmol} / \mathrm{L}$, TG 1.5 $\mathrm{mmol} / \mathrm{L}$, LDL-C $5.9 \mathrm{mmol} / \mathrm{L}$ and HDL-C $1.15 \mathrm{mmol} / \mathrm{L}$ (21).

Fibrates alter the size distribution of LDL particles towards large, less dense and less atherogenic particles. Levels of IDL are also reduced, accounting for the efficacy of fibrates in familial dysbetalipoproteinaemia (apoE2 homozygosity or type III hyperlipidaemia). (16)

Fenofibrate monotherapy in combined hyperlipidaemia with mild hypertriglyceridaemia (HTG)

A 12 week study of $160 \mathrm{mg} /$ day fenofibrate therapy in 187 patients with mixed hyperlipidaemia whose baseline lipid levels were TC $6.7 \mathrm{mmol} / \mathrm{L}$, TG 3.1 $\mathrm{mmol} / \mathrm{L}, \mathrm{HDL}-\mathrm{C} 1.1 \mathrm{mmol} / \mathrm{L}$ and LDL-C $4.1 \mathrm{mmol} / \mathrm{L}$ resulted in 5.5\% LDL-C reduction overall (30). This response was reduced in those with LDL-C $<4.1$ $\mathrm{mmol} / \mathrm{L}$, those with $\mathrm{HDL}-\mathrm{C}<1.0 \mathrm{mmol} / \mathrm{L}$, diabetic patients, and men vs. women. In patients with baseline $T G=<3.1 \mathrm{mmol} / \mathrm{L}$, $\mathrm{LDL}-\mathrm{C}$ was reduced by 
$9.9 \%$ compared with $1.1 \%$ reduction in those with baseline $\mathrm{TG}>3.1 \mathrm{mmol} / \mathrm{L}$ (the median TG was $3.1 \mathrm{mmol} / \mathrm{L})(30)$.

Fenofibrate monotherapy in moderate HTG

When fenofibrate was given to patients with baseline TG 4-5.6 $\mathrm{mmol} / \mathrm{L}$ over an eight week period, TG levels decreased by $46 \%$ and LDL-C levels increased by $14.5 \%$. (17).

Fenofibrate monotherapy in severe HTG

When fenofibrate was given to patients with TG at baseline $5.6-16.9 \mathrm{mmol} / \mathrm{L}$, TG levels decreased by $55 \%$ and LDL-C levels increased by $45 \%$. (17).

Fenofibrate combination therapy with statins

Fibrate therapy may be effective as second-line add-on therapy in patients whose LDL-C levels remain above target in spite of statin and/or ezetimibe therapy. The first-line choice for addition to statin therapy is ezetimibe, as it has a predictable effect in lowering LDL-C ( 20\%), and is well tolerated. (22) Ezetimibe therapy results in smaller effects on plasma lipid levels other than LDL-C.

In the SAFARI trial, simvastatin $20 \mathrm{mg} /$ day plus fenofibrate $160 \mathrm{mg} /$ day was compared with simvastatin alone in 619 patients with combined hyperlipidaemia over a 12 week period (Table 5). (23) Compared with statin monotherapy, statin-fibrate therapy increased HDL-C and apoA-1 levels and decreased levels of TG, LDL-C, non-HDL-C and apoB (Table 4). There were 
no cases of clinical myopathy or rhabdomyolysis (23). With fenofibrate therapy, the proportion of large LDL particles increased from $9.2 \%$ at baseline to $28.6 \%$, and the proportion of small dense LDL particles decreased from $72.2 \%$ at baseline to $32.1 \%(23)$.

Table 4. Effects on plasma lipids of fenofibrate and simvastatin in combination vs. simvastatin monotherapy.

\begin{tabular}{|l|l|l|l|}
\hline Lipid & Baseline mean & $\begin{array}{l}\% \\
\text { simvastatin 20mg }\end{array}$ & $\begin{array}{l}\% \text { change } \\
\text { simvastatin 20mg } \\
+ \text { fenofibrate }\end{array}$ \\
\hline HDL-C & $1.14^{*}$ & 10 & 18 \\
\hline LDL-C & $4.22^{*}$ & -26 & -31 \\
\hline TG & $2.91^{*}$ (median) & -20 & -43 \\
\hline Non-HDL-C & $5.52^{*}$ & -26 & -35 \\
\hline apoA-1 & $149 \#$ & 5 & 9 \\
\hline apoB & $165 \#$ & -23 & -33 \\
\hline
\end{tabular}

HDL-C, high-density lipoprotein cholesterol; LDL-C, low-density lipoprotein cholesterol;

TG, triglycerides

- $\mathrm{mmol} / \mathrm{L}$

\# mg/dl

Fenofibrate and ezetimibe monotherapy vs. combination therapy in hyperlipidaemia with mild HTG 
Fenofibrate therapy in combination with ezetimibe was compared with ezetimibe and fenofibrate monotherapy in 625 patients with mixed hyperlipidaemia. Baseline lipid levels were similar in each group (see Table 1), comprising placebo $(N=61)$, ezetimibe alone $(N=173)$, fenofibrate alone $(\mathrm{N}=179)$ and fenofibrate plus ezetimibe $(\mathrm{N}=175)$ (30). LDL-C levels were reduced by $13.4 \%$ overall with ezetimibe monotherapy ( $\mathrm{P}<0.001$ vs. $5.5 \%$ reduction with fenofibrate monotherapy) and by $20.4 \%$ with combination therapy ( $p<0.001$ vs. ezetimibe and fenofibrate monotherapy). In patients with baseline $\mathrm{TG}=<3.1 \mathrm{mmol} / \mathrm{L}$, LDL-C was reduced by $15.3 \%$ and $27.8 \%$ with ezetimibe and combination therapy respectively $(p<0.001)$, compared with $11.5 \%$ and $12.9 \%$ reduction with ezetimibe and combination therapy respectively in those with baseline $T G>3.1 \mathrm{mmol} / \mathrm{L}$ (NS) (30). HDL-C was significantly increased and triglycerides significantly decreased by fenofibrate alone or the combination compared with placebo $(p<0.001)$ without difference between these two treatment groups. Combination therapy increased HDL-C by $19 \%$, and reduced LDL-C, non-HDL-C and triglycerides by $20.4 \%, 30.4 \%$ and $44 \%$, respectively. The study concluded that fenofibrate and fenofibrate plus ezetimibe have similar effects on the lipid profile, other than a 2-fold greater percentage reduction in TC with combination therapy (30). The ezetimibe product information, however, warns that combination therapy with fibrates can result in 1.5 fold increase in ezetimibe plasma levels, but no clinical adverse events were experienced in the above study (31). A subsequent study showed further benefit from fenofibrate combined with simvastatin/ezetimibe (32). 
In other trials, fenofibrate-statin therapy had limited additional effects on plasma lipid levels compared with statin monotherapy (24). In one trial of 304 subjects, after 12-18 weeks of therapy the extra LDL-C reduction was 4.54.8\%. In 364 diabetic patients the extra HDL-C increase was 4.8-5.2\%. In 104 diabetic subjects the change in non-HDL-C was $-1.8 \%$ (NS) and the change in TC/HDL-C ratio $-2.7 \%$ (NS). In 154 diabetic patients the extra reduction in TG was $-13.6 \%$.

More patients reach NCEP ATP-III targets for LDL-C with combination statinfibrate therapy compared with statin monotherapy alone. In two trials of 240 evaluable patients with diabetes, 170 reached targets. One trial of 80 subjects over 24 weeks compared atorvastatin $20 \mathrm{mg}$ with atorvastatin $20 \mathrm{mg}$ plus fenofibrate $200 \mathrm{mg} /$ day. The odds ratio for reaching target favoured combination therapy (OR 9.75, $\mathrm{Cl} 1.16-82.11)$. The other trial of 163 subjects over 18 weeks compared rosuvastatin $40 \mathrm{mg}$ with rosuvastatin $40 \mathrm{mg}$ plus fenofibrate $200 \mathrm{mg} /$ day. The odds ratio was non-significant at $0.5(\mathrm{Cl} 0.20$ 1.24), probably reflecting the more potent effect on lowering LDL-C of rosuvastatin $40 \mathrm{mg}$ than atorvastatin $20 \mathrm{mg}(24)$.

\section{The FIELD and ACCORD studies}

The large FIELD and ACCORD studies of fenofibrate in diabetic patients did not show significant reductions in LDL-C levels compared with placebo. FIELD was a study of 9,795 asymptomatic diabetic patients randomised for 5 years to placebo vs fenofibrate therapy, with additional therapy at the discretion of the treating physician (28). After 4 months there was a $12 \%$ 
$(0.4 \mathrm{mmol} / \mathrm{L})$ reduction in LDL-C levels in the fenofibrate vs placebo group, and after 3 years there was no difference. The data was difficult to interpret because of a significant proportion of drop-ins with statin therapy, which was greater in the placebo group than the fenofibrate group. The ACCORD study treated 5,518 diabetic patients (one-third of whom had CAD) for 4.7 years with either fenofibrate or placebo, on a background of open-label simvastatin therapy (29). Levels of LDL-C did not change significantly in the fenofibratetreated group, and were not significantly different from the placebo-treated group (29). The data from these large trials suggest there may be less reduction of LDL-C with fibrates in diabetic patients compared with nondiabetic patients, but other causes may also be involved such as differences in baseline levels and whether or not statins are used.

Niacin as an alternative to fenofibrate for add-on therapy with statins

An alternative first-line add-on therapy with statins is niacin. The advantage of niacin is that it decreases LDL-C more than fibrates, has a greater effect on raising HDL-C, and also lowers Lp (a) levels (25). The problem is that niacin is so poorly tolerated. Probably less than $10 \%$ of patients can tolerate 3000mg per day for an extended period.

The lack of clinical outcomes data, such as results of the AIM-HIGH study, however, has to be considered (26). AIM-HIGH was a five-year study of almost 3500 patients with low HDL-C, designed to examine whether raising HDL using extended-release niacin would be beneficial. The trial was stopped 17 months prematurely on the grounds of "futility" because high-dose, 
extended-release niacin offered no benefits beyond statin therapy alone in reducing cardiovascular-related complications. There was no evidence that this would change by continuing the trial. Use of niacin with statins now depends on results of HPS2-THRIVE, a large clinical outcomes trial in which extended-release niacin in combination with simvastatin and laropiprant (an inhibitor of the niacin receptor responsible for skin flushing) is compared with placebo and simvastatin therapy (27).

Other combination therapy with statins

Other therapies which effectively lower LDL-C as add-on to statins include plant sterols, 2-4 g/day, and bile sequestrants (4-20g/day).

Recommendation 4, (Grade A). Fenofibrate is preferred to gemfibrozil for combination therapy with statins in order to reduce the likelihood of adverse effects on skeletal muscle.

Fibrate monotherapy is not frequently associated with myalgia, although the incidence may be increased with concomitant statin therapy, especially gemfibrozil (33-39). Gemfibrozil inhibits glucuronyl transferase, one of the enzymes involved in the liver's excretion pathways for statins. The enzyme catalyses attachment of a glucuronyl ring to statin molecules and facilitates their hepatic uptake and excretion into bile. When administered with gemfibrozil, blood levels of statins may be considerably raised because of increased Cmax, time/concentration area under the curve (AUC), and $t 1 / 2$ of 
the statin or its active metabolites (33). The AUC with gemfibrozil plus statin is two- to fourfold greater than with a statin alone. The higher the statin blood level, the greater the risk of myopathy and rhabdomyolysis. In contrast to gemfibrozil, fenofibrate does not inhibit hepatic glucuronyl transferase, and does not result in significant elevation in plasma levels of statins nor have any significant effect on the incidence of muscular side effects $(37,38)$.

Recommendation 5, (Grade B). Fibrates are appropriate for prevention of macrovascular cardiovascular disease in diabetic and non-diabetic patients with fasting triglyceride levels of $2.3 \mathrm{mmol} / \mathrm{L}$ or more and/or low HDL-C levels less than $1.0 \mathrm{mmol} / \mathrm{L}$ in men and less than $1.2 \mathrm{mmol} / \mathrm{L}$ in women, assuming adequate glycaemic control and exclusion of other secondary causes of dyslipidaemia.

High TG, small dense LDL particles, and low HDL-C commonly occur together as the "atherogenic triad", particularly in states of insulin resistance such as obesity, the metabolic syndrome, and diabetes mellitus. Fibrates are particularly effective in treating the atherogenic triad, and several large trials of fibrates have shown reduction in CAD events, which is mainly confined to patients with the atherogenic triad in both diabetic and non-diabetic patients.

The three most important outcome trials were VA-HIT, Field and LIPIDACCORD trials. In the VA-HIT trial of 2531 men with CHD the baseline triglyceride level was $1.7 \mathrm{mmol} / \mathrm{L}$ and on Gemfibrozil $1200 \mathrm{mg}$ per day it decreased to $1.3 \mathrm{mmol} / \mathrm{L}(\mathrm{P}<0.001)$. HDL-C increased from $0.8 \mathrm{mmol} / \mathrm{L}$ by $6 \%$. 
The authors attributed the $11 \%$ reduction in CHD events over 5.1 years to the increase of HDL not the triglyceride reduction.

A recent meta-analysis of several of these trials investigated the effect of fibrates on the incidence of nonfatal myocardial infarction (MI) and all-cause mortality using random effects models (5) Compared with placebo, fibrates were associated with greater reductions in TC (range: -2.6 to $-0.1 \mathrm{mmol} / \mathrm{L}$ ) and TG (range: -3.63 to $-0.24 \mathrm{mmol} / \mathrm{L}$ ), and a greater increase in $\mathrm{HDL}-\mathrm{C}$ (range: +0.03 to $+0.46 \mathrm{mmol} / \mathrm{L}$ ) in all trials. Fibrates tended to be associated with a greater reduction in LDL-C (range: -1.98 to $-1.03 \mathrm{mmol} / \mathrm{L}$ ) than placebo, although these changes were not consistent across all trials. Fibrates reduced the rate of nonfatal $\mathrm{MI}$ (odds ratio $=0.78 ; 95 \% \mathrm{Cl}, 0.69-0.89$ ), but not all-cause mortality (odds ratio $=1.05 ; 95 \% \mathrm{Cl}, 0.95-1.15$ )

It was concluded that fibrates improve lipid profiles and are associated with decreased nonfatal MI, but have no substantial effect on all-cause mortality. It was suggested that clinical use of fibrates include treatment for patients with statin resistance or isolated hypertriglyceridemia (HTG), or as an adjunct to other lipid-lowering therapies (5). Reduction in CAD risk would appear to be greater if fibrates were reserved for patients with atherogenic dyslipidaemia (high TG and low HDL-C) (5). Similar conclusions were reached in another meta-analysis (40).

Fig. 1 shows the results of a meta-analysis of reduction of risk of main coronary events in patients of all ages treated with fibrates (1). The reduction in major coronary events was significant in the two studies using gemfibrozil 
(VA-HIT and Helsinki Heart Study), and only trended toward significant in the other fibrate trials. It was concluded that fibrates may have a benefit in select patients in whom statins are not an option and when HDL or TG modification is indicated (1).

Fig 1. Meta-analysis of reduction of risk of main coronary events in patients of all ages treated with fibrates (1).

\section{Staty}

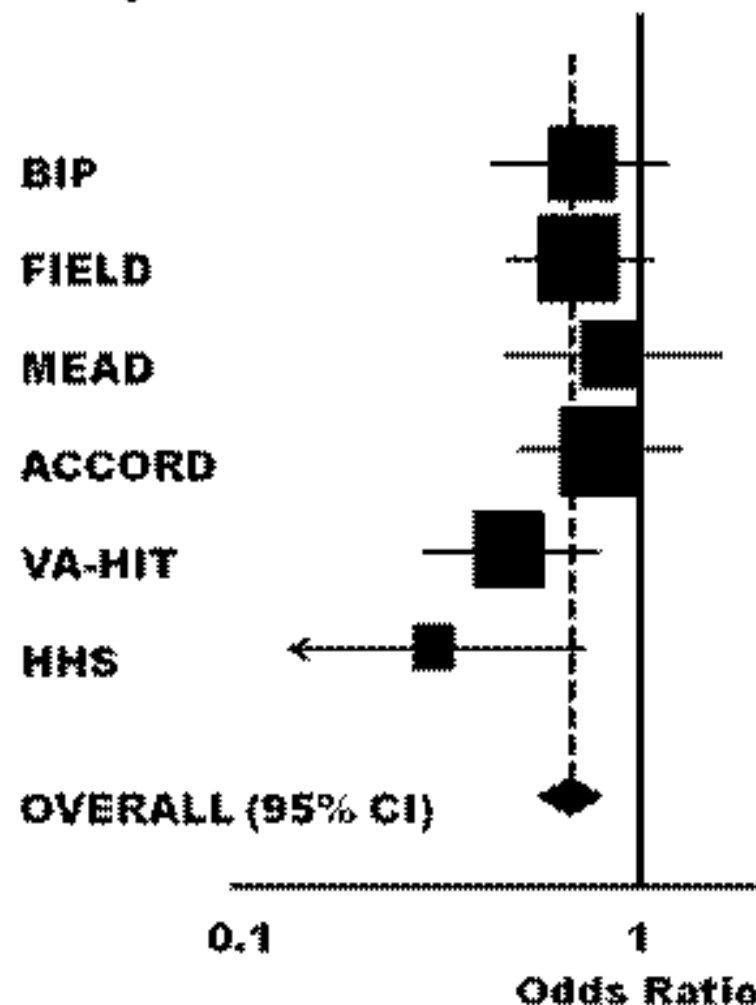

Odds Ratio $(95 \% \mathrm{Cl})$
$\%$

Weingt
$0.89(0,73,1.0$. 16$) \quad 16.8$

$0.88(0.74,1.05) \quad 22.8$

$0.95(0.95,1.19) \quad 13.0$

$0.93(0.78,1,10) \quad 23.7$

$0.76(0.62,0.92) \quad 17.6$

$0.64(0.46,0.96) \quad$ (.6.

$0.86(0.79 .0 .94)$ tho

In the FIELD study, individuals with marked dyslipidaemia (elevated triglycerides $\geq 2.3 \mathrm{mmol} / \mathrm{L}$ and low $\mathrm{HDL}$ cholesterol $(<1.0 \mathrm{mmol} / \mathrm{L}$ in men and $<1.3 \mathrm{mmol} / \mathrm{L}$ in women) were at the highest risk of CVD (17.8\% over 5 years) (41-46). A $27 \%$ relative risk reduction $(95 \% \mathrm{Cl} 9-42, \mathrm{P}=0.005$; number needed to treat $=23$ ) was observed in this group. The absolute risk reduction 
in the presence of marked dyslipidaemia was $4.3 \%$ (from 17.8 to $13.5 \%$ ), compared with $0.8 \%$ (from 13.0 to $12.2 \%$ ) in its absence, when the number needed to treat was 143 . The results of the FIELD subgroup analysis of patients with marked dyslipidaemia are supported by findings from other fibrate trials, including the Bezafibrate Infarct Prevention (BIP) study, the HHS, the Veterans Administration High-Density Lipoprotein Intervention Trial Intervention Trial and the ACCORD-Lipid study. Nevertheless, because all results were presented with $P$ values unadjusted for multiple comparisons, these findings should be regarded as hypothesis-generating, therefore are given a Grade C recommendation (4).

Patients with the metabolic syndrome (MetS) also benefited from fenofibrate therapy in the FIELD study (47) Those with MetS according to ATPIII criteria and treated with placebo had a CVD event rate of $14.5 \%$ over the 5 years of the study, compared with a rate of $13.1 \%$ in those treated with placebo $(11 \%$ relative risk reduction, hazard ratio $0.89,95 \% \mathrm{Cl} 0.79-1.00, \mathrm{p}=0.052)$. Those without MetS according to ATPIII criteria and treated with placebo had a CVD event rate of $11.3 \%$ over the 5 years of the study, compared with a rate of $9.7 \%$ in those treated with placebo $(12 \%$ relative risk reduction, hazard ratio $0.88,95 \% \mathrm{Cl} 0.65-1.19, \mathrm{p}=0.375)$. The treatment benefit of fenofibrate therapy was $17 \%$ in those with MetS and no previous CVD, compared with a benefit of $1 \%$ in those with previous CVD. The treatment effect of fenofibrate was also greater in women (18\% risk reduction) compared with men $(7 \%$, NS). Fenofibrate independently reduced CVD events compared with placebo 
in patients with hypertension and low HDL-C levels. The respective hazard ratios were $0.88(\mathrm{Cl} 0.79-0.99, \mathrm{p}=0.34)$ and $0.86(0.75-0.99, \mathrm{p}=0.03)(47)$.

Recommendation 6 (Grade B). Fenofibrate may be considered for prevention of microvascular disease (retinopathy, sensory neuropathy, nephropathy and to prevent amputations) in patients with type 2 diabetes mellitus, assuming adequate glycaemic control and exclusion of other secondary causes of dyslipidaemia.

Diabetic microvascular disease (DMVD) is a complication of diabetes that affects small vessels of the retina, kidneys, peripheral nerves and feet, and is partly responsible for progression of retinopathy, nephropathy, neuropathy and peripheral vascular disease (PVD).

Improvement in DMVD with fibrate therapy was first reported in the FIELD study, which showed lower rates of laser therapy for diabetic proliferative retinopathy (DPR) (42). Similar but even more pronounced benefits for diabetic retinopathy (DR) were observed on the ACCORD-EYE substudy, which differed in several respects from the FIELD study in design and reporting characteristics $(48,49)$. Using a standard ophthalmological scale, progression of DR in ACCORD-Eye required $\geq 3$ step progression in both eyes, while 1 or 2 step progression in the worst eye sufficed in FIELD. Preexisting DR occurred in $50 \%$ and $20 \%$ of patients, and the duration of type 2 diabetes was 10 years and 5 years in ACCORD-Eye and FIELD respectively. In ACCORD-EYE there was a $40 \%$ lower rate of laser therapy in the 
fenofibrate-simvastatin treatment group (6.5\% over 4 years) compared with the placebo-simvastatin group (10.2\%, adjusted OR $0.6,95 \% \mathrm{Cl} 0.42-0.87$, $p=0.006)$. In contrast to this significant improvement, effects of statin therapy on DR progression have been inconsistent $(48,49)$. As suggested by the results of the FIELD study, much of the benefit for DR in ACCORD-EYE is likely to be due to fenofibrate therapy rather than to combination statin-fibrate therapy.

In the FIELD study non-traumatic amputations for DMVD were also reduced in the fenofibrate group compared with the placebo group, in spite of increased statin drop-ins in the placebo group (43-46). There were 115 amputations related to DM, with the risk of first amputations being reduced from 70 events in the placebo group to 45 events in the fenofibrate group (HR $0.64,95 \% \mathrm{Cl}$ 0.44-0.94, $\mathrm{p}=0.02$ ). Minor amputations (below the ankle) in patients without known large-vessel disease occurred in 34 patients treated with placebo and in 18 patients treated with fenofibrate $(\mathrm{HR} 0.53, \mathrm{Cl} 0.30$ 0.94, $\mathrm{p}=0.027$ ). No difference in risk was observed for major amputations (above the ankle), HR 0.93 (24 vs 26 events), $\mathrm{Cl} 0.53-1.62, \mathrm{p}=0.79$. The benefits of fenofibrate were independent of diabetes control, the presence of dyslipidaemia, and use of drugs acting on the renin-angiotensin system.

Previous studies of statins, vitamin E, antihypertensive agents and aspirin failed to reduce amputations in people with diabetes, indicating that fenofibrate (and possibly fibrates as a class) may have a unique beneficial action in this regard $(44,45)$. Fenofibrate could therefore become part of 
standard care for high-risk patients (those with previous CVD, previous nontraumatic amputation, previous skin ulceration, smoking, long duration of diabetes, tall height, large-vessel peripheral vascular disease, or recognised DMVD).

Renal microvascular disease is detected and quantified by measurement of urinary albumin concentrations (50). In non-diabetic patients, significant proteinuria is present when the urinary albumin: creatinine ratio (ACR) is 30 $\mathrm{mg} / \mathrm{mmol}$ or more (equivalent to a protein excretion of $0.5 \mathrm{~g} / 24 \mathrm{~h}$ or more) $(50)$. In diabetic patients, microalbuminuria is clinically significant if ACR $>2.5$ $\mathrm{mg} / \mathrm{mmol}$ in men and $>3.5 \mathrm{mg} / \mathrm{mmol}$ in women (50). In the FIELD study, reduced estimated GFR (eGFR) and albuminuria were independent risk factors for cardiovascular events and mortality rates. Albuminuria increased CVD risk, with microalbuminuria and macroalbuminuria increasing total CVD (HR 1.25 [1.01-1.54] and 1.19 [0.76-1.85], respectively; CVD risk was further modified by renal status changes over the first 2 years. In multivariable analysis, $77 \%$ of the effect of eGFR and $81 \%$ of the effect of albumin: creatinine ratio was accounted for by other variables, principally low HDLcholesterol and elevated blood pressure (51).

The FIELD study of diabetic patients showed significant reduction in the rate of progression of normoalbuminuria (NA) to microalbuminuria (MiA) or from MiA to macroalbuminuria $(\mathrm{MaA})$ in the fenofibrate-treated group $(10 \%)$ compared with the placebo-treated group (11\%) (55) Regression of albuminuria occurred in $9 \%$ and $8 \%$ of patients respectively. There was 
therefore a difference of $2.6 \%$ patients either not progressing or regressing in the fenofibrate group $(p=0.002)$. The number of patients requiring dialysis was also lower in the fenofibrate group vs placebo group (16 vs. 21).

Fenofibrate reduced albuminuria and slowed estimated GFR loss over 5 years, despite initially and reversibly increasing plasma creatinine. During fenofibrate run-in, plasma creatinine increased by $10.0 \mu \mathrm{mol} / \mathrm{l}(\mathrm{p}<0.001)$, but quickly reversed on placebo assignment. It remained higher on fenofibrate than on placebo, but the chronic rise was slower (1.62 vs $1.89 \mu \mathrm{mol} / \mathrm{I}$ annually, $p=0.01$ ), with less estimated GFR loss (1.19 vs $2.03 \mathrm{ml} \mathrm{min}(-1)$ $1.73 \mathrm{~m}(-2)$ annually, $\mathrm{p}<0.001)$.

After washout, estimated GFR had fallen less from baseline on fenofibrate $(1.9 \mathrm{ml} \min (-1) 1.73 \mathrm{~m}(-2), \mathrm{p}=0.065)$ than on placebo $(6.9 \mathrm{ml} \min (-1) 1.73$ $\mathrm{m}(-2), \mathrm{p}<0.001)$, sparing $5.0 \mathrm{ml} \min (-1) 1.73 \mathrm{~m}(-2)(95 \% \mathrm{Cl} 2.3-7.7, \mathrm{p}<$ 0.001). Greater preservation of estimated GFR with fenofibrate was observed with baseline hypertriglyceridaemia ( $n=169$ vs 491 without) alone, or combined with low HDL-cholesterol ( $\mathrm{n}=140$ vs 520 without) and reductions of $\geq 0.48 \mathrm{mmol} / \mathrm{l}$ in triglyceride over the active run-in period (pre-randomisation) ( $n=356$ vs 303 without).

Fenofibrate reduced urine albumin concentrations and hence albumin/creatinine ratio by $24 \%$ vs $11 \%$ ( $p<0.001$; mean difference $14 \%$ [95\% Cl 9-18]; $p<0.001$ ), with $14 \%$ less progression and $18 \%$ more 
albuminuria regression $(p<0.001)$ than in participants on placebo. End-stage renal event frequency was similar $(n=21$ vs $26, p=0.48)$.

The FIELD study therefore suggested fenofibrate may delay albuminuria and GFR impairment in type 2 diabetes patients. (28)

Reduced progression of albuminuria was also observed with fenofibrate therapy in the DAIS trial (52). In the ACCORD trial, post-randomisation MiA occurred in $38.2 \%$ and $41.6 \%$ of patients treated with fenofibrate and placebo respectively $(p=0.01)$ (51). Post-randomisation MaA occurred in $10.5 \%$ and $12.3 \%$ of patients treated with fenofibrate and placebo respectively $(p=0.03)$.

The possible mechanisms by which microavascular protection may occur with fenofibrate therapy include improved endothelial-dependent vascular reactivity, anti-inflammatory effects with reduction in pro-inflammatory markers (interleukin-6, interleukin 1- beta, tumour necrosis factor alpha), reduced plasma viscosity, improved insulin sensitivity, protection from ischaemia, reduction in apoptosis, increased nitric oxide synthesis, and neuroprotective effects $(43,44)$.

\section{Other issues}

Uniform agreement of the panel was not always reached on a number of issues, reflecting the varied opinions of experts and consensus panels elsewhere and the need for further research to provide more robust data from which to draw conclusions. One such area relates to cut-off levels for HDL-C and TG for which fibrate therapy is indicated to control residual risk (the risk of 
CVD remaining in spite of statin therapy). Some authorities suggest appropriate TG levels are $>2.2 \mathrm{mmol} / \mathrm{L}$, others $>2.0 \mathrm{mmol} / \mathrm{L}$ as in the CSANZ /Heart Foundation of Australia Lipid position statement (58). Cut-points are, however, arbitrary and driven by retrospective analyses of subgroups in fibrate trials. No prospective trial has yet been performed with these cut-points as inclusion criteria, although such a trial for fenofibrate was recently recommended by the FDA to confirm subgroup analysis of the ACCORD-Lipid trial showing benefit in dyslipidaemic diabetic patients (29). We have given grade $B$ to recommendation 5 for these reasons.

Cut-offs for HDL-C are also arbitrary and also reflect subgroup analyses of fibrate trials. Fibrates increase HDL-C levels, especially in those with low baseline levels. This was shown in a large study of 7,098 patients with dyslipidaemia, treated with placebo or micronised fenofibrate $200 \mathrm{mg}$ daily (bioequivalent to fenofibrate $160 \mathrm{mg}$ tablets) for 24 weeks (47). The increase in HDL-C levels was inversely related to baseline levels, being $+22.7 \%$ overall, $+90.2 \%$ (lowest quintile, baseline HDL-C levels $<0.65 \mathrm{mmol} / \mathrm{L}$, mean increasing from 0.54 to $1.00 \mathrm{mmol} / \mathrm{L}$ ), $+58.3 \%$ (second lowest quintile, baseline HDL-C $<0.77 \mathrm{mmol} / \mathrm{L}$, mean increasing from 0.66 to $1.02 \mathrm{mmol} / \mathrm{L}$ ), $+44.3 \%$ (middle quintile, baseline HDL-C $<0.9 \mathrm{mmol} / \mathrm{L}$, mean increasing from 0.76 to $1.07 \mathrm{mmol} /$ ), $+36.1 \%$ (second highest quintile, baseline HDL-C <1.03 $\mathrm{mmol} / \mathrm{L}$, mean increasing from 0.84 to $1.02 \mathrm{mmol} / \mathrm{L}$ ), $+31.6 \%$ (highest quintile, baseline HDL-C levels $<1.16 \mathrm{mmol} / \mathrm{L}$, mean increasing from 0.89 to $1.16 \mathrm{mmol} / \mathrm{L})$. All HDL-C levels in the fenofibrate group were higher than placebo $(p<0.0001)$. 
The effect of fenofibrate on HDL-C was also greatest among high-risk patients with low levels of HDL-C (53). These changes were higher than those reported in the clinical outcomes trials with fibrates, and most studies show increases in HDL-C between $5-15 \%$. Elevation of HDL-C appears to be blunted in diabetic patients, in whom the functionality of HDL may also be impaired.

Fibrates increase HDL-C levels by several mechanisms. Exchange of TG in VLDL and LDL for cholesteryl esters in HDL via CETP is reduced, as a result of reduced TG secretion in VLDL (54). Fibrates enhance transcription of hepatic apoA-I, thereby increasing apoA-I-mediated HDL production. Fibrates also activate the liver $\mathrm{X}$ receptor and through increased PPARa activity promote synthesis of ABCA-I, which delivers free cholesterol from cell membranes to nascent HDL particles in the blood (54).

Low levels of HDL-C are generally associated with high TG levels in patients with insulin resistance, therefore are frequently encountered in diabetes, the metabolic syndrome and central obesity. The combination of high TG and low HDL-C is regularly associated with small-sized LDL particles and increased apoB-100-containing lipoproteins, constituting the "atherogenic lipid triad". Patients with HTG of other causes also have low HDL-C, particularly in severe HTG in which levels of HDL-C may be as low as $0.2 \mathrm{mmol} / \mathrm{L}$. In the absence of HTG, such low levels of HDL-C suggest a genetic cause with a mutation 
either in the apoA-1, ABCA1 or LCAT gene. Such patients respond poorly (if at all) to currently available lipid modifying therapy.

Levels of HDL-C are remarkably stable in the majority of patients and in spite of therapy only fluctuate by small amounts in contrast to TG and LDL-C levels; nevertheless such small changes powerfully predict changes in CVD risk according to epidemiological data and analyses of clinical trials with statins and fibrates in which part of the observed benefit has been attributed to increases in HDL-C levels.

Low levels of HDL-C may therefore be targeted in patients on statin therapy in order to improve residual risk, in much the same way that elevated TG levels are also targeted. Because disturbances in HDL-C and TG occur together, it is debatable as to whether there is benefit to be gained by targeting one lipoprotein vs. the other. The answer to this question will be provided by results of trials with drugs that specifically raise HDL-C and have little impact on other lipoprotein classes. The cholesterol ester transfer protein (CETP) inhibitors may closest to such as class, although they also lower LDL-C and TG levels. Studies with recombinant HDL have suggested that targeting HDLC alone in patients with acute coronary syndrome (not necessarily with low HDL-C levels) is likely to be beneficial, as rapid regression of coronary atherosclerosis has been observed (55). This is currently an area of intensive research. 
In the meantime, fibrates and niacin are reasonably effective in raising HDL-C levels. Omega-3 fatty acids may result in similar improvement in patients with HTG (56). In general, the lower the HDL-C level, the greater in the percentage increase, although achieving target HDL-C levels (> $1.0 \mathrm{mmol} / \mathrm{L})$ in patients with baseline levels $<0.5 \mathrm{mmol} / \mathrm{L}$ remains problematic. The effectiveness of lipid modifying drugs in raising HDL-C is shown in Table 5.

Table 5. Effectiveness of lipid modifying drugs in raising HDL-C levels (percentage increase from baseline)

CETP inhibitors

Fibrates

Statins

Resins

Plant sterols

Ezetimibe

Omega-3 fatty acids
$55-120 \%+$

$5-25 \%$

$5-15 \%$

$3-5 \%$

$2-3 \%$

$5-10 \%$

up to $5 \%$

There is considerable variation in reported effects of fibrates and other drugs on raising HDL-C in clinical trials and in individual patients. This is not unexpected, given the complexity of $\mathrm{HDL}-\mathrm{C}$ metabolism and its interaction with other plasma lipoproteins. 


\section{Conclusions}

The European Medicines Agency recently recommended fibrates (including fenofibrate, ciprofibrate, etofibrate, bezafibrate and gemfibrozil) as second-line therapy for patients with dyslipidaemia, with the exception of patients with severe HTG and patients who cannot tolerate statins where they are first-line (57). The rationale for this recommendation was that benefits with fibrate therapy continue to outweigh risk in the treatment of lipid disorders. The recommendation endorsed a previous one in 2005 by the EMA's Pharmacovigilance Working Party. A specific recommendation was provided for fenofibrate to be used with statins if inadequate lipid control has been achieved with statin monotherapy.

Outcome studies of fibrates in combination with non-statin lipid modifying drugs are yet to be performed and are unlikely to be performed in the near future, given the efficacy of statin monotherapy and the limited extra benefits conferred (to date) by additional agents. The CETP inhibitors may prove to be the exception to the rule, but outcome studies of CETP inhibitors in combination with fibrates have yet to be instituted. There remains considerable scope for future investigation of a variety of lipid modifying drugs, alone and in combination, on CV and microvascular disease outcomes and their potential mechanisms of benefit.

The relationship of baseline lipids to microvascular benefits of fenofibrate in diabetic subjects is currently under intense investigation as are the 
mechanisms for benefit. It seems likely that multiple biochemical pathways are involved, reflecting the complex gene effects of PPAR-alpha agonism. Microvascular benefits of fibrates other than fenofibrate may also occur, as these drugs share a common mechanism of action. Publications on these topics from the FIELD and ACCORD study investigators are anticipated in the near future. These, as well as results of current trials in progress (e.g. DALoutcomes, HPS2-THRIVE) will impact on future use of fibrates and other lipid modifying agents in clinical practice. A post-statin era may yet evolve with more effective means of prevention through new ways of targeting plasma lipoproteins and other risk factors.

\section{Potential conflicts of interest}

$\mathrm{IHC}$ is a member of the Australian Lipid Advisory Boards of AstraZeneca, Abbott and Merck, Sharp and Dohme (Australia). He has received research grants, honoraria and travel grants from AstraZeneca, Abbott, Merck, Sharp and Dohme, Pfizer, Novartis, Servier, Sanofi-Aventis and Bristol-Myers Squibb.

KMK is a member of the Australian Lipid Advisory Boards of Pfizer, Abbott and Merck, Sharp and Dohme and has received honoraria and travel grants from AstraZeneca, Abbott, Merck, Sharp and Dohme, Pfizer, Novartis, Servier, Sanofi-Aventis and Bristol-Myers Squibb.

SW and DC have no potential conflicts to declare. 


\section{References.}

1. Streja D, Streja E. management of dyslipidemia in the elderly. http://www.endotext.org/aging/aging4/agingframe4.htm

2. Bays H, Stein EA. Expert Opin Pharmacother 2003 Nov, 4 (11): 1901-38 Pharmacotherapy for dyslipidaemia--current therapies and future agents.

3. Alagona P. Vasc Health Risk Management. 2010 May 25, 6;351-62. Fenofibric acid: a new fibrate approved for use in combination with statin for the treatment of mixed dyslipidemia.

4. Lee M, Saver JL, Towfighi A. Atherosclerosis 2011 Aug; 21(2): 492-8. Epub 2011 Ap 27. Efficacy of fibrates for cardiovascular risk reduction in persons with atherogenic dyslipidaemia: a meta-analysis.

5. Abourbih S, Filion KB, Joseph L. Am J Med. 2009 Oct;122(10):962. e1-8, Epub 2009 Aug 19. Effect of fibrates on lipid profiles and cardiovascular outcomes: a systematic review.

6. Barter PJ, Rye KA. Arterioscler Thromb Vasc Biol. 2008 Jan;28(1):39-46. Epub 2007 Aug 23. Is there a role for fibrates in the management of dyslipidemia in the metabolic syndrome?

7. Bruckert E, Labreuche J, Deplanque D. J Cardiovasc Pharmacol. 2011 Feb;57(2):267-72. Fibrates effect on cardiovascular risk is greater in patients with high triglyceride levels or atherogenic dyslipidemia profile: a systematic review and meta-analysis. 
8. Rubenfire M, Brook RD, Rosenson RS. Am J Med. 2010 Oct;123(10):892-

8. Treating mixed hyperlipidemia and the atherogenic lipid phenotype for prevention of cardiovascular events.

9. Farnier M. Vasc Health Risk Manag. 2008;4(5):991-1000. Update on the clinical utility of fenofibrate in mixed dyslipidemias: mechanisms of action and rational prescribing.

10. Hokanson JE, Austin MA. J Cardiovasc Risk 1996 Apr;3(2):213-19. Plasma triglyceride level is a risk factor for cardiovascular disease independent of high-density lipoprotein cholesterol level: a meta-analysis of population-based prospective studies.

11. Patel A, Barzi F, Jamrozik K. Circulation 2004 Oct 26;110(17):267886.Epub 2004 Oct 18. Serum triglycerides as a risk factor for cardiovascular diseases in the Asia-Pacific region.

12. Mazza A, Tikhonoff V, Schiavon L. Intern Med J. 2005 Oct;35:604-610. Triglycerides + high-density-lipoprotein-cholesterol dyslipidaemia, a coronary risk factor in elderly women: the CArdiovascular STudy in the ELderly.

13. Sarwar N, Danesh J, Eiriksdottir G, Circulation 2007 Jan 30;115(4):45058. Epub 2006 Dec 26. Triglycerides and the risk of coronary heart disease: 10,158 incident cases among 262,525 participants in 29 Western prospective studies. 
14. Miller M, Stone NJ, Ballantyne C, Circulation 2011 May 24;123(20):2292333. Epub 2011 apr 18. Triglycerides and cardiovascular disease: a scientific statement from the American Heart Association.

15. Watts GF, Karpe F. Heart 2011 Mar;97(5):350-56. Triglycerides and atherogenic dyslipidaemia: extending treatment beyond statins in the high-risk cardiovascular patient.

16. Chapman MJ, Ginsberg HN, Amarenco P. Eur Heart J. 2011 Jun; 32(11): 1345-61. Epub 2011 Apr 29. Triglyceride-rish lipoproteins and high-density lipoprotein cholesterol in patients at high risk of cardiovascular disease: evidence and guidance for management.

17. Abbott Pharmaceuticals website: www.rxabbott.com , accessed 1/12/11

18. Reiner Z, Catapano AL, De Backer G. Eur Heart J. 2011 Jul;32(14):1769818. Epub 2011 June 28. European Association for Cardiovascular Prevention \& Rehabilitation, ESC Committee for Practice Guidelines (CPG) 2008-2010 and 2010-2012 Committees, Bax J, Vahanian A, Auricchio A, et al. ESC/EAS Guidelines for the management of dyslipidaemias: the Task Force for the management of dyslipidaemias of the European Society of Cardiology (ESC) and the European Atherosclerosis Society (EAS).

19. Miller M, Stone NJ, Ballantyne C. Circulation. 2011 May 24;123(20):2292333. American Heart Association Clinical Lipidology, Thrombosis, and Prevention Committee of the Council on Nutrition, Physical Activity, and Metabolism; Council on Arteriosclerosis, Thrombosis and Vascular Biology; Council on Cardiovascular Nursing; Council on the Kidney in Cardiovascular 
Disease. Triglycerides and cardiovascular disease: a scientific statement from the American Heart Association.

20. Watts GF, Sullivan DR, Poplawski N. Atheroscler Suppl. 2011 Sep 12. Familial Hypercholesterolaemia: A model of care for Australasia.

21. Product information for fenofibrate www.rxabbott.com. Accessed 1/12/11.

22. Hamilton-Craig I, Kostner K, Colquhoun D. Vascular Health \& Risk Management. 2010 Nov 10;6:1023-37. Combination therapy of statin and ezetimibe for treatment of familial hypercholesterolaemia.

23. Grundy SM, Vega GL, Yuan Z, Am J Cardiol 2005 Feb 15;95(4):462-8. Effectiveness and tolerability of simvastatin plus fenofibrate for combined hyperlipidemia (the SAFARI trial).

24. Moutzouri E, Kei A, Elisaf MS. Vasc health Risk management. 2010 Aug 9;6:525-39. Management of dyslipidemias with fibrates, alone and in combination with statins: role of delayed-release fenofibric acid.

25. Kostner K, Gupta S. Expert Opinion on Pharmacother. 2008 Nov;9(16): 2911-20. Niacin: A lipid polypill?

26. NIH pulls plug on AIM-HIGH trial with niacin. http://www.theheart.org/article/1231453.do Accessed 3/7/11. 
27. A Randomized Trial of the Long-term Clinical Effects of Raising HDL Cholesterol With Extended Release Niacin/Laropiprant Available at: HPS2THRIVEhttp://clinicaltrials.gov/ct2/show/NCT00461630?term=HPS2+thrive\&ra $n k=1$. Accessed 3/7/11

28. Davis TM, Ting R, Best JD. Diabetologia 2011 Feb;54(2):280-90. Epub 2010 Nov 4. Effects of fenofibrate on renal function in patients with type 2 diabetes mellitus: The Fenofibrate Intervention and Event Lowering in Diabetes (FIELD) Study.

$29 \mathrm{H}$ Ginsberg. Effects of combination lipid therapy on cardiovascular events in type 2 diabetes mellitus: the action to control cardiovascular risk in diabetes (ACCORD) Lipid Trial Diabetes Care. 2011 May;34 Suppl 2:S107-8

30. Farnier M, Freeman M, Macdonell G. Eur Heart J. 2005 May;26(9):897905. Epub 2005 Mar 21. Efficacy and safety of the coadministration of Ezetimibe with fenofibrate in patients with mixed hyperlipidaemia.

31. Product information for ezetimibe. http://www.zetia.com/ezetimibe/zetia/hcp/product_information/pi/index.jsp> Accessed 1/12/11.

32. Farnier M, Roth E, Gil-Extremera B, Am Heart J. 2007 Feb; 153(2):335. e1-8. Efficacy and safety of the coadministration of ezetimibe/simvastatin with fenofibrate in patients with mixed hyperlipidemia. 
33. Backman JT, Kyrklund C, Kivisto KT. Clin Pharmacol Ther. 2000 Aug; 68(2):122-9. Plasma concentrations of active simvastatin acid are increased by gemfibrozil.

34. Kyrklund C, Backman JT, Kivisto KT. Clin Pharmacol Ther. 2001 May; 69(5):340-45. Plasma concentrations of active lovastatin acid are markedly increased by gemfibrozil but not by bezafibrate.

35. Kyrklund C, Backman JT, Neuvonen M, Clin Pharmacol Ther. 2003 Jun; 73(6):538-544. Gemfibrozil increases plasma pravastatin concentrations and reduces pravastatin renal clearance.

36. Schneck DW, Birmingham BK, Zalikowski JA. Clin Pharmacol Ther. 2004 May; 75(5):455-63. The effect of gemfibrozil on the pharmacokinetics of rosuvastatin.

37. Bergman AJ, Murphy G, Burke J. J Clin Pharmacol. 2004 Sep; 44(9):1054-62. Simvastatin does not have a clinically significant pharmacokinetic interaction with fenofibrate in humans.

38. Pan WJ, Gustavson LE, Achari R. J Clin Pharmacol. 2000 Mar; 40(3):316-23. Lack of a clinically significant pharmacokinetic interaction between fenofibrate and pravastatin in healthy volunteers. 
39. Bottorff MB Am J Cardiol. 2006 Apr 17;97(8A):27C-31C. Epub 2006 Jan 25. Statin safety and drug interactions: clinical implications.

40. Jun M, Foote C, Lv J. Lancet 2010 May 29; 375(9729): 1875-84. Epub 2010 May 10. Effects of fibrates on cardiovascular outcomes: a systematic review and meta-analysis.

41. Keech A, Simes RJ, Barter P, Lancet. 2005 Nov 26;366(9500):1849-61. Effects of long-term fenofibrate therapy on cardiovascular events in 9795 people with type 2 diabetes mellitus (the FIELD study): randomised controlled trial.

42. Keech AC, Mitchell P, Summanen PA. Lancet 2007 Nov 17;370(9600):1687-97. Epub 2007 Nov 7. Effect of fenofibrate on the need for laser treatment for diabetic retinopathy (FIELD study): a randomised controlled trial.

43. Robins SJ, Bloomfield HE. Current Opinion Lipidology. 2006 Aug;17(4):431-39. Fibric acid derivatives in cardiovascular disease prevention: results from the large clinical trials.

44. Rajamani K, Colman PG, Li LP. Lancet. 2009 May 23;373(9677):178088). Effect of fenofibrate on amputation events in people with type 2 diabetes 
mellitus (FIELD study): a prespecified analysis of a randomised controlled trial.

45. Fazio S, Linton MF. Lancet 2009 May 23;373(9677):1740-41. Fenofibrate and risk of minor amputations in diabetes.

46. Studer M, Briel M, Leimenstoll B. Arch Intern Med. 2005 Apr 11; 165(7):725-30. Effect of different antilipidemic agents and diets on mortality: A systematic review.

47. Scott R, O'Brien R, Fulcher G, Pardy C, D'Emden M, Tse D, Taskinen MR, Ehnholm C, Keech A. Diabetes Care. 2009 Mar; 32(3):493-8. Effects of fenofibrate treatment on cardiovascular disease risk in 9,795 individuals with type 2 diabetes and various components of the metabolic syndrome: the Fenofibrate Intervention and Event Lowering in Diebetes (FIELD) study.

48. The ACCORD Study Group and ACCORD Eye Study group. Effects of medical therapies on retinopathy progression in type 2 diabetes. NEJM 2010. DOI: 10.1056/NEJMoa1001288, accessed 20 June 2011.

49. Klein B. NEngl J M. 2010 Jul 15;363(3):287-88. Epub 2010 Jun 29. DOI: 10.1056/NEJMe1005667, accessed 20 June 2011. Reduction in risk of progression of diabetic retinopathy. 
50. NICE clinical guideline 73. Chronic kidney disease: Early identification and management of chronic kidney disease in adults in primary and secondary care. 2008.

51: Drury PL, Ting R, Zannino D. Diabetologia. 2011 Jan;54(1):32-43. Epub 2010 Jul 30. Estimated glomerular filtration rate and albuminuria are independent predictors of cardiovascular events and death in type 2 diabetes mellitus: the Fenofibrate Intervention and Event Lowering in Diabetes (FIELD) study.

52. Lancet. 2001 Mar 24; 357(9260):905-10. Effect of fenofibrate on progression of coronary-artery disease in type 2 diabetes: the Diabetes Atherosclerosis Intervention Study, a randomised study.

53. Després JP. Am J Cardiol. 2001 Dec 20;88(12A):30N-36N. Increasing high-density lipoprotein cholesterol: an update on fenofibrate.

54. Kostner KM, Kostner GM. Pharazie Unserer Zeit. 2007;36(2): 114-9. Fibrate bei Lipidstoffwechselstoerungen. (Fibrate in lipid metabolism disorders).

55. Kostner KM, Edmund Cauza. Future Cardiology. 2005 Nov;1(6) 767-73. HDL therapy: the next big step in the treatment of atherosclerosis? 
56. Goldberg RB, Sabharwal AK. Curr Opin Endocrinol Diabetes Obes. 2008 Apr;15(2):167-74. Fish oil in the treatment of dyslipidemia.

57. European Medicines Agency press release 22 Oct 2010, www.ema.europa.eu. Accessed 1/12/11.

58. Tonkin A, Barter P, Best J, Boyden A, Furler J, Hossack K, et al. National Heart Foundation of Australia; Cardiac Society of Australia and New Zealand. Heart Lung Circ. 2005 Dec;14(4):275-91. National Heart Foundation of Australia and the Cardiac Society of Australia and New Zealand: position statement on lipid management--2005. 
